Bull. Korean Math. Soc. 46 (2009), No. 3, pp. 489-498

DOI 10.4134/BKMS.2009.46.3.489

\title{
ON SEPARATIVE REFINEMENT MONOIDS
}

\author{
HuANYin Chen
}

\begin{abstract}
We obtain two new characterizations of separativity of refinement monoids, in terms of comparability-type conditions. As applications, we get several equivalent conditions of separativity for exchange ideals.
\end{abstract}

A commutative monoid $(M,+, 0)$ has a refinement (or is a refinement monoid) if, for all $a, b, c$ and $d$ in $M$, the equation $a+b=c+d$ implies the existence of $a_{1}, b_{1}, c_{1}, d_{1} \in M$ such that $a=a_{1}+d_{1}, b=b_{1}+c_{1}, c=a_{1}+b_{1}$ and $d=d_{1}+c_{1}$.

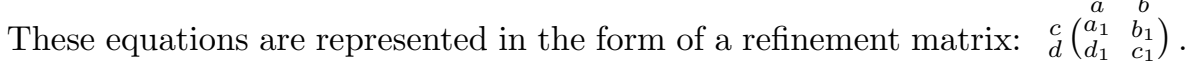
Refinement monoids have been extensively studied in recent years (cf. [4] and [7]). A commutative monoid $M$ is separative if, for all $a, b \in M, 2 a=a+b=2 b$ implies $a=b$. Separativity is a weak form of cancellativity for commutative monoids. Many authors have studied separative refinement monoids from various view-points (see [3-4] and [6-7]). In this article, we get two new characterizations of separative refinement monoids. We prove that every separative refinement monoid can be characterized by a certain sort of comparability. Also we introduce the concept of refinement extensions of a refinement monoid. We see that every separative refinement monoid can be characterized by such refinement extensions. Let $I$ be an ideal of a ring $R$. We use $F P(I)$ to denote the class of finitely generated projective right $R$-modules $P$ with $P=P I$ and $V(I)$ to denote the monoid of isomorphism classes of objects from $F P(I)$. Following Ara et al. (see [3]), an exchange ideal $I$ of a $\operatorname{ring} R$ is separative if $V(I)$ is a separative refinement monoid, that is, for any $A, B, C \in F P(I)$, $A \oplus A \cong A \oplus B \cong B \oplus B \Longrightarrow A \cong B$. We say that $R$ is a separative ring if $R$ is separative as an ideal of $R$.

Separativity plays a key role in the direct sum decomposition theory of exchange rings. It seems rather likely that non-separative exchange rings should exist. We say that an exchange ring $R$ satisfies the comparability axiom provided that, for any finitely generated projective right $R$-modules $A$ and $B$, either $A \lesssim^{\oplus} B$ or $B \lesssim^{\oplus} A$. In [7, Theorem 3.9], Pardo showed that every

Received June 13, 2008; Revised January 9, 2009.

2000 Mathematics Subject Classification. 20M14, 16E50.

Key words and phrases. refinement monoid, separativity, exchange ideal. 
exchange ring satisfying the comparability axiom is separative. But the converse is not true. For instance, there exist unit-regular rings which do not satisfy the comparability axiom (see [5, Example 8.7]). We will give a new characterization of the separativity for exchange ideals of a ring, in terms of comparability-type conditions. Using refinement extensions of a refinement monoid, we observe that the separativity over exchange ideals possesses nice weak cancellation properties for arbitrary right modules.

Throughout, all monoids are commutative, so we will write + for the monoid operation and 0 for the identity elements of all monoids. Every monoid $M$ will be endowed with the preordering $\leq$ defined by $a \leq b$ in $M$ if and only if there exists some $c \in M$ such that $a+c=b$. A monoid $M$ has an order-unit $u$ if $u \in M$ is an element such that every element of $M$ is bounded above by a positive multiple of $u$. A subclass $I$ of a monoid $M$ is an o-ideal provided that $(\forall x, y \in I \Longleftrightarrow x+y \in I)$. All rings in this article are associative with identities and all modules are right unitary modules. Let $A$ and $B$ be right $R$-modules. The symbol $A \lesssim^{\oplus} B$ means that $A$ is isomorphic to a direct summand of $B$ and $n A$ means that the direct sum of $n$ copies of $A$. We always use $\mathbb{N}$ to denote the set of all positive integers.

For refinement monoids $M$, we note that separativity can be reduced to the statement $(\forall a, b, c \in M)(a+c=b+c$ with $c \leq a, b \Longrightarrow a=b)$. In general, this property is weaker than separativity. This follows very easily from $[3$, Lemma 2.1].

Theorem 1. Let $M$ be a refinement monoid. Then the following are equivalent:

(1) $M$ is separative.

(2) $(\forall a, b \in M)(a=2 b$ and $3 a=3 b \Longrightarrow a \leq b$ or $b \leq a)$.

(3) $(\forall a, b, c \in M)(c+a=c+b$ with $c \leq a, b \Longrightarrow a \leq b$ or $b \leq a)$.

Proof. $(1) \Rightarrow(2)$ is clear by [3, Lemma 2.1].

$(2) \Rightarrow(3)$ Given $c+a=c+b$ with $c \leq a, b$ in $M$, then there exist $e, f \in M$ such that $a=c+e$ and $b=c+f$; hence, $2 a=a+(c+e)=(b+c)+e=b+a$. Likewise, we have $2 b=a+b$. This implies that $2 a=2 b$. Furthermore, we get $3 a=a+2 b=(a+b)+b=3 b$. So either $a \leq b$ or $b \leq a$.

$(3) \Rightarrow(1)$ Suppose that $c+a=c+b$ with $c \leq a, b$. It will suffice to show that $a=b$. In view of [3, Lemma 2.7], we have a refinement matrix over $M: \begin{array}{cc}a \\ c\end{array}\left(\begin{array}{cc}b & c \\ d_{1} & a_{1} \\ b_{1} & c_{1}\end{array}\right)$, where $c_{1} \leq a_{1}, b_{1}$. From $a_{1}+c_{1}=b_{1}+c_{1}$ with $c_{1} \leq a_{1}, b_{1}$, we deduce that $a_{1} \leq b_{1}$ or $b_{1} \leq a_{1}$. If $a_{1} \leq b_{1}$, then $b_{1}=a_{1}+e$. Thus $c=c_{1}+b_{1}=c_{1}+a_{1}+e=c+e$. As $c \leq a, b$, we have $a=a+e$ and $b=b+e$; hence, $a=a+e=a_{1}+d_{1}+e=b_{1}+d_{1}=b$. The proof of the case $b_{1} \leq a_{1}$ is just symmetric from the case $a_{1} \leq b_{1}$. By the note above, we obtain the result.

Corollary 2. Let $M$ be a refinement monoid. Then the following are equivalent: 
(1) $M$ is separative.

(2) $(\forall a, b \in M)(\forall n \in \mathbb{N})(n a=n b$ and $(n+1) a=(n+1) b \Longrightarrow a \leq b$ or $b \leq a)$.

(3) $(\forall a, b \in M)(2 a=a+b=2 b \Longrightarrow a \leq b$ or $b \leq a)$.

Proof. $(1) \Rightarrow(2)$ is obvious by [3, Lemma 2.1].

$(2) \Rightarrow(3)$ Suppose that $2 a=a+b=2 b$ in $M$. Then $2 a=2 b$ and $3 a=$ $a+(a+b)=2 a+b=3 b$. Furthermore, we get $4 a=3 b+a=2 b+(a+b)=4 b$. Similarly, we deduce that $n a=n b$ and $(n+1) a=(n+1) b(n \geq 2)$. So $a \leq b$ or $b \leq a$.

$(3) \Rightarrow(1)$ Given $c+a=c+b$ with $c \leq a, b$ in $M$, then we have $e, f \in M$ such that $a=c+e$ and $b=c+f$. It is easy to check that $a+a=a+(c+e)=$ $(b+c)+e=b+a$. Similarly, $b+b=a+b$. Hence $a \leq b$ or $b \leq a$. By virtue of Theorem 1 , the result follows.

We say that $M$ is an ordered-separative monoid provided that $(\forall a, b \in$ $M)(a+b=2 b \Longrightarrow a \leq b)$. In [8, Theorem 4.1], Wehrung proved that if $M$ is separative, then $M$ is order-separative. We note that 'separativity' used in [8] differs from that in this paper. Wehrung's 'separativity' satisfies an additional condition: $(\forall a, b, c \in M)(a+c \leq b+c$ with $c \propto b \Rightarrow a \leq b)$. A natural problem asked whether the converse is true. In general, order-separativity does not imply general separativity. Let $M$ be the monoid generated by three elements $a, b$ and $c$ such that $2 a=0, a+b=c, a+c=b$ and $b+c=2 b$. Then $M=\{0, a, b, c, 2 b, 3 b, 4 b, \ldots\}$ defined by the following addition:

\begin{tabular}{c|cccc}
+ & 0 & $a$ & $b$ & $c$ \\
\hline 0 & 0 & $a$ & $b$ & $c$ \\
$a$ & $a$ & 0 & $c$ & $b$ \\
$b$ & $b$ & $c$ & $2 b$ & $2 b$ \\
$c$ & $c$ & $b$ & $2 b$ & $2 b$
\end{tabular}

and $a+m b=m b, c+m b=(m+1) b(m \geq 2)$. As $2 b=b+c=2 c, M$ is not a separative monoid. But one checks that $M$ is an ordered-separative monoid. In [4, Proposition 9.5], Brookfield proved that every refinement order-separative monoid is separative. Now we generalize Brookfield's result as follows.

Corollary 3. Let $M$ be a refinement monoid. If $(\forall a, b \in M)(a+b=2 b \Longrightarrow$ $a \leq b$ or $b \leq a)$, then $M$ is separative.

Proof. It is obvious from Corollary 2.

The converse of Corollary 3 is not true. Let $\{0, \infty\}$ be the monoid such that $\infty+\infty=\infty$, and let $\mathbb{R}^{++}$the subgroup of strictly positive real numbers. Let $M$ be the monoid obtained from $\{0, \infty\} \times \mathbb{R}^{++}$by adding a zero element. Since $\{0, \infty\}$ and $\mathbb{R}^{++}$are separative refinement subgroups, we prove that $M$ is a separative refinement monoid. Choose $a=(0,1)$ and $b=(\infty, 1)$. Then $a+b=2 b$, while $a \not \leq b$ and $b \not \leq a$. 
Following Ara (cf. [1-2]), an ideal $I$ of a ring $R$ is an exchange ideal provided that for every $x \in I$ there exist an idempotent $e \in I$ and elements $r, s \in I$ such that $e=x r=x+s-x s$. Let $I$ be an exchange ideal of a ring $R$, and let $e \in R$ be an idempotent. By [1, Lemma 1.1], one easily checks that $e I e$ is an exchange ring.

Lemma 4. Let $I$ be an exchange ideal of a ring $R$. Then for all right $R$ modules $A, B, C, D$ such that $A \oplus B \cong C \oplus D$ and $A \in F P(I)$, there are right $R$-modules $A_{1} \cong A_{2}, B_{1} \cong B_{2}, C_{1} \cong C_{2}, D_{1} \cong D_{2}$ such that $A=A_{1} \oplus D_{1}, B=$ $B_{1} \oplus C_{1}, C=A_{2} \oplus B_{2}$, and $D=D_{2} \oplus C_{2}$.

Proof. Suppose that $\psi: A \oplus B \cong C \oplus D$. Then $A \oplus B=\psi^{-1}(C) \oplus \psi^{-1}(D)$. As $A \in F P(I)$, there is a right $R$-module $E$ such that $A \oplus E \cong n R$ for some $n \in \mathbb{N}$. Let $e: n R \rightarrow A$ be the projection onto $A$. Then $A \cong e(n R)$, whence $\operatorname{End}_{R}(A) \cong e M_{n}(R) e$. As $A=A I$, we have $e(n R)=e(n R) I \subseteq n I$. Set $e=$ $\left(\alpha_{1}, \ldots, \alpha_{1}\right) \in M_{n}(R)$. Then $e(1,0, \ldots, 0)^{T} \in n I$; hence, $\alpha_{1} \in n \bar{I}$. Likewise, we have $\alpha_{2}, \ldots, \alpha_{n} \in n I$. It follows that $e \in M_{n}(I)$. Since $I$ is an exchange ideal of $R, M_{n}(I)$ is also an exchange ideal of $M_{n}(R)$, and then $\operatorname{End}_{R}(A)$ is an exchange ring. Thus $A$ has the finitely exchange property. So we can find $B_{1} \subseteq \psi^{-1}(C)$ and $B_{2} \subseteq \psi^{-1}(D)$ such that $A \oplus B=A \oplus B_{1} \oplus B_{2}$. So $B \cong B_{1} \oplus B_{2}$. As $B_{1} \subseteq \psi^{-1}(C) \subseteq B_{1} \oplus\left(A \oplus B_{2}\right)$, we get $\psi^{-1}(C)=\psi^{-1}(C) \bigcap\left(B_{1} \oplus A \oplus B_{2}\right)=B_{1} \oplus$ $\psi^{-1}(C) \bigcap\left(A \oplus B_{2}\right)$. Let $C_{1}=\psi(C) \bigcap\left(A \oplus B_{2}\right)$. Then $C \cong \psi^{-1}(C)=B_{1} \oplus C_{1}$, Likewise, we have a right $R$-module $D_{1}$ such that $D \cong \psi^{-1}(D)=B_{2} \oplus D_{1}$. In addition, $A \oplus\left(B_{1} \oplus B_{2}\right)=A \oplus B=\psi^{-1}(C) \oplus \psi^{-1}(D)=\left(B_{1} \oplus C_{1}\right) \oplus\left(B_{2} \oplus D_{1}\right)$; hence, $A \cong C_{1} \oplus D_{1}$. Therefore we complete the proof.

Theorem 5. Let $I$ be an exchange ideal of a ring $R$. Then the following are equivalent:

(1) I is separative.

(2) For any $A, B \in F P(I), C \oplus A \cong C \oplus B$ with $C \lesssim \oplus A, B \Longrightarrow A \lesssim \oplus B$ or $B \lesssim \oplus A$.

(3) For any $A, B \in F P(I), 2 A=2 B$ and $3 A=3 B \Longrightarrow A \lesssim \oplus B$ or $B \lesssim \oplus A$.

(4) For any $A, B \in F P(I), 2 A \cong A \oplus B \cong 2 B \Longrightarrow A \lesssim^{\oplus} B$ or $B \lesssim^{\oplus} A$.

Proof. In view of Lemma $4, V(I)$ is a refinement monoid. Applying Theorem 1 and Corollary 2 to $V(I)$, we obtain the result.

Corollary 6. Let $I$ be an exchange ideal of a ring $R$, and let $m, n \geq 2$ with $\operatorname{gcd}(m, n)=1$. Then the following are equivalent:

(1) $I$ is separative.

(2) For any $A, B \in F P(I), m A \cong m B$ and $n A \cong n B \Longrightarrow A \lesssim^{\oplus} B$ or $B \lesssim \oplus A$.

Proof. $(1) \Rightarrow(2)$ Since $I$ is separative, $V(I)$ is a separative monoid. According to [4, Proposition 8.10], we get $A \cong B$, as desired. 
$(2) \Rightarrow(1)$ Given $2 A \cong A \oplus B \cong 2 B$ with $A, B \in F P(I)$, then $m A \cong m B$ and $n A \cong n B$ by an easy induction. So either $A \lesssim^{\oplus} B$ or $B \lesssim^{\oplus} A$. In view of Theorem $5, I$ is separative.

So far, we have been investigating separativity only in a refinement monoid. Let $M$ be a refinement submonoid of a monoid $N$. We say that $N$ is a refinement extension of $M$ in case the following hold:

(1) $M$ is an o-ideal of $N$.

(2) $(\forall b, c, d \in N)(\forall a \in M)(a+b=c+d \Longrightarrow$ there exists a refinement matrix over $N: \begin{array}{cc}c \\ d\end{array}\left(\begin{array}{cc}a & b \\ a_{1} & b_{1} \\ d_{1} & c_{1}\end{array}\right)$.

We write $\{0,1, \infty\}$ for the monoid such that $1+1=1+\infty=\infty+\infty=\infty$. Since the equation $1+1=\infty+\infty$ can not be refined, $\{0,1, \infty\}$ is not a refinement monoid. The monoid $\{0, \infty\}$ is a refinement submonoid of $\{0,1, \infty\}$. Obviously, the condition (1) is equivalent to the statement: $(\forall a \in M)(b \leq a$ in $N \Longrightarrow b \in M)$. Although the condition (2) is satisfied in this case, we claim that $\{0,1, \infty\}$ is not a refinement extension of $\{0, \infty\}$. This is clear from $1 \leq \infty$ and $1 \notin\{0, \infty\}$. Let $\mathbb{Z}^{+}$be the monoid of non-negative integers, and let $A=\{0,2,3,4, \ldots\}$ be the submonoid of $\mathbb{Z}^{+}$obtained by deleting the number 1. Let $N=A \times\{0, \infty\}$ and $M=0 \times\{0, \infty\}$. Then $N$ is a refinement extension of the refinement $M$, while $N$ is not a refinement monoid. As $(2,0) \not 3(3,0)$ in $N$, we see that $(2,0)+(4,0)=(3,0)+(3,0)$ has no a refinement matrix over $N$. Now we observe that separativity can be partially extended to refinement extensions of a refinement monoid.

Theorem 7. Let $N$ be a refinement extension of a refinement monoid $M$. Then the following are equivalent:

(1) $M$ is separative.

(2) $(\forall a, b \in N)(\forall c \in M)(c+a=c+b$ with $c \leq a, b \Longrightarrow a=b)$.

(3) $(\forall a, b \in N)(\forall c \in M)(2 c+a=2 c+b \Longrightarrow c+a=c+b)$.

Proof. (1) $\Rightarrow(2)$ Suppose that $c \in M, a, b \in N$ such that $c+a=c+b$ with $c \leq a, b$. Since $N$ is a refinement extension of $M$, we have a refinement matrix over $\left.N: \begin{array}{cc}c & c \\ c & a \\ c_{1} & a_{1} \\ b_{1} & d_{1}\end{array}\right)$. From $c_{1} \leq c \leq a=a_{1}+d_{1}$ in $N$, there exists some $e \in N$ such that $c_{1}+e=a_{1}+d_{1}$. As $c_{1} \leq c$ and $c \in M$, we deduce that

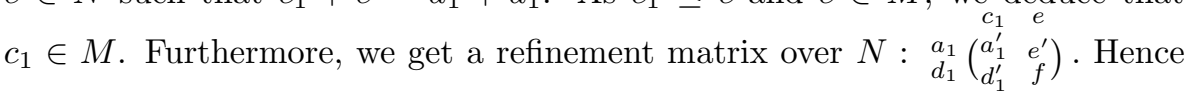
$c_{1}=a_{1}^{\prime}+d_{1}^{\prime}$ and $a_{1}^{\prime} \leq a_{1}, d_{1}^{\prime} \leq d_{1}$. So we can write that $d_{1_{c}}=d_{1}^{\prime}+d_{1}^{\prime \prime}$ for some $d_{1}^{\prime \prime} \in N$. Thus there is a refinement matrix over $N: \underset{b}{c}\left(\begin{array}{cc}c & a_{1}^{\prime} \\ b_{1}+d_{1}^{\prime} & a_{1}+d_{1}^{\prime} \\ d_{1}^{\prime \prime}\end{array}\right)$. Let $c_{2}=a_{1}^{\prime}, a_{2}=a_{1}+d_{1}^{\prime}, b_{2}=b_{1}+d_{1}^{\prime}$ and $d_{2}=d_{1}^{\prime \prime}$. We get a refinement matrix over $N$ :

$$
\begin{gathered}
c \\
c \\
b
\end{gathered}\left(\begin{array}{cc}
c_{2} & a_{2} \\
b_{2} & d_{2}
\end{array}\right)
$$


with $c_{2} \leq a_{2}$. In addition, we see that $c_{2} \leq c_{1}$ and $b_{1} \leq b_{2}$. As $c \leq b$, we apply the argument above to the refinement matrix $(*)$ and get a new refinement matrix over $\left.N: \begin{array}{cc}c & a \\ c & c_{3} \\ b_{3} & d_{3}\end{array}\right)$ with $c_{3} \leq b_{3}$. Furthermore, we have $c_{3} \leq c_{2} \leq a_{2} \leq a_{3}$. Thus $c_{3}+a_{3}=c_{3}+b_{3}=c \in M$ with $c_{3} \leq a_{3}, b_{3}$. Clearly, $c_{3}, a_{3}, b_{3} \in M$. As $M$ is a separative monoid, it follows that $a_{3}=b_{3}$. Therefore $a=a_{3}+d_{3}=b_{3}+d_{3}=b$, as desired.

$(2) \Rightarrow(3)$ Suppose that $c \in M$ and $a, b \in N$ such that $2 c+a=2 c+b$. Then $c+(c+a)=c+(c+b)$ with $c \leq c+a, c+b$; hence, $c+a=c+b$.

$(3) \Rightarrow(1)$ is trivial by [3, Lemma 2.1].

Let $a$ and $b$ be elements in a monoid. The notation $a \propto b$ means that $a \leq n b$ for some $n \in \mathbb{N}$.

Corollary 8. Let $N$ be a refinement extension of a refinement monoid $M$. Then the following are equivalent:

(1) $M$ is separative.

(2) $(\forall a, b \in N)(\forall c \in M)(c+a=c+b$ with $c \propto a, b \Longrightarrow a=b)$.

Proof. (1) $\Rightarrow$ (2) Suppose that $c+a=c+b$ and $c \in M, c \propto a, b$. Then we may choose $n \in \mathbb{N}$ such that $c \leq n a, n b$. So there exists $d \in N$ such that $c+d=a+(n-1) a$. Since $N$ is a refinement extension of $M$, we have a refinement matrix over $N: \begin{gathered}a \\ (n-1) a\end{gathered}\left(\begin{array}{cc}c & d \\ c_{1} & d_{1} \\ a_{1} & e_{1}\end{array}\right)$. This infers that $a_{1}+e_{1}=a+(n-2) a$. It follows by $a_{1} \leq c \in M$ that $a_{1} \in M$. Similarly, we have a refinement matrix over $\left.N: \underset{c}{a} \begin{array}{cc}a_{1} & e_{1} \\ c_{2} & d_{2} \\ a_{2} & e_{2}\end{array}\right)$. Furthermore, we have a refinement matrix

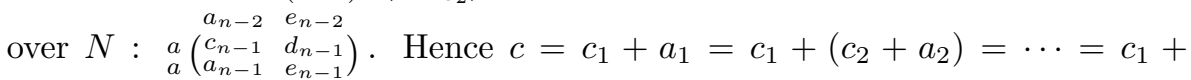
$c_{2}+\cdots+c_{n-1}+a_{n-1}$. Set $c_{n}=a_{n-1}$. Then $c=c_{1}+c_{2}+\cdots+c_{n}$ with $c_{1}, \ldots, c_{n} \leq a$. As $c_{1} \leq c \in M$, we see that $c_{1} \in M$. Similarly, we prove that $c_{1}=c_{11}+\cdots+c_{1 m_{1}}$ with $c_{1 j} \leq b\left(j=1, \ldots, m_{1}\right)$. Analogously, we have $c_{i j} \in M$ such that $c_{i}=c_{i 1}+\cdots+c_{i m_{i}}(i=2, \ldots, n)$. As a result, $\left(\sum_{1 \leq i \leq n, 1 \leq j \leq m_{i}} c_{i j}\right)+a=\left(\sum_{1 \leq i \leq n, 1 \leq j \leq m_{i}} c_{i j}\right)+b$ with all $c_{i j} \in M, c_{i j} \leq a, b$. By using Theorem 7 repeatedly, we get $a=b$, as required.

$(2) \Rightarrow(1)$ is obvious by [3, Lemma 2.1].

Recall that a right $R$-module $P$ is a $R$-progenerator in case there exist $m, n \in$ $\mathbb{N}$ and modules $P^{\prime}$ and $R^{\prime}$ such that $m R \cong P \oplus P^{\prime}$ and $n P \cong R \oplus R^{\prime}$. Let $I$ be a separative exchange ideal of a ring $R$, and let $C$ be a finitely generated projective right $R$-module with $C=C I$. If $A$ and $B$ are any $R$-progenerators such that $C \oplus A \cong C \oplus B$, we claim that $A \cong B$. This is an immediate consequence of Corollary 8.

Theorem 9. Let $I$ be an exchange ideal of a ring $R$. Then the following are equivalent:

(1) I is separative. 
(2) For any $C \in F P(I), C \oplus A \cong C \oplus B$ with $C \lesssim \oplus A, B \Longrightarrow A \cong B$ for any right $R$-modules $A$ and $B$.

Proof. (1) $\Rightarrow(2)$ Let $\mathcal{M}_{R}$ denote the class of all right $R$-modules, and let $W(R)$ be the monoid of isomorphism classes of objects from $\mathcal{M}_{R}$. Then $V(I)$ is a submonoid of $W(R)$. Suppose that $C \oplus A \cong C \oplus B$ with $C \in F P(I)$ and $A, B \in \mathcal{M}_{R}$. According to Lemma 4, we have a refinement matrix $\begin{gathered}C \\ B\end{gathered}\left(\begin{array}{cc}C & A \\ C_{1} & A_{1} \\ B_{1} & D_{1}\end{array}\right)$ over $W(R)$. This means that $W(R)$ is a refinement extension of the refinement monoid $V(I)$. It follows by Theorem 7 that $A \cong B$.

$(2) \Rightarrow(1)$ For any $A, B, C \in F P(I), C \oplus A \cong C \oplus B$ with $C \lesssim^{\oplus} A, B \Longrightarrow$ $A \cong B$, and therefore the result follows from [3, Lemma 2.1].

Corollary 10. Let $A$ be a finitely generated projective right module over a separative exchange ring $R$. If $A$ and $B$ are any right $R$-modules such that $C \oplus A \cong C \oplus B$ with $C \lesssim^{\oplus} A, B$, then $A \cong B$.

Proof. It is obvious by Theorem 9 .

Theorem 11. Let $N$ be a refinement extension of a refinement monoid $M$. If $N$ contains an order-unit $u$, then the following are equivalent:

(1) $M$ is separative.

(2) $(\forall a, b \in N)(\forall c \in M)(c+a=c+b \leq u$ with $c \leq a, b \Longrightarrow a \leq b$ or $b \leq a)$.

(3) $(\forall a, b, c \in M)(c+a=c+b \leq u$ with $c \leq a, b \Longrightarrow a \leq b$ or $b \leq a)$.

Proof. $(1) \Rightarrow(2)$ is obvious by Theorem 7 .

$(2) \Rightarrow(3)$ is trivial.

$(3) \Rightarrow(1)$ Given $c+a=c+b$ with $c \leq a, b$ in $M$, then we can find some $n \in \mathbb{N}$ such that $c \leq n u$ in $N$. Since $N$ is a refinement extension of $M$, by induction, the refinement property also holds for the sum $n u$. So there exist $c_{1}, \ldots, c_{n} \leq u$ such that $c=c_{1}+\cdots+c_{n}$. Hence $c_{1}+\left(c_{2}+\cdots+c_{n}+a\right)=c_{1}+\left(c_{2}+\cdots+c_{n}+b\right)$. Let $a_{1}=c_{2}+\cdots+c_{n}+a$ and $b_{1}=c_{2}+\cdots+c_{n}+b$. Then $c_{1}+a_{1}=c_{1}+b_{1}$ with $c_{1} \in M$ and $c_{1} \leq a_{1}, b_{1}$. By the proof of Theorem 7, we have a refinement matrix over $N: \begin{array}{cc}c_{1} \\ b_{1}\end{array}\left(\begin{array}{cc}c_{1}^{\prime} & a_{1}^{\prime} \\ b_{1}^{\prime} & d_{1}\end{array}\right)$, where $c_{1}^{\prime} \leq a_{1}^{\prime}, b_{1}^{\prime}$. It follows from $c_{1}^{\prime}+a_{1}^{\prime}=c_{1}^{\prime}+b_{1}^{\prime}=c_{1} \leq u$ with $c_{1}^{\prime} \leq a_{1}^{\prime}, b_{1}^{\prime}$ that either $a_{1}^{\prime} \leq b_{1}^{\prime}$ or $b_{1}^{\prime} \leq a_{1}^{\prime}$. If $a_{1}^{\prime} \leq b_{1}^{\prime}$, then $b_{1}^{\prime}=a_{1}^{\prime}+e$. As a result, we get $c_{1}=c_{1}^{\prime}+b_{1}^{\prime}=c_{1}^{\prime}+a_{1}^{\prime}+e=c_{1}+e$. Since $c_{1} \leq a_{1}, b_{1}$, we see that $a_{1}=a_{1}+e$ and $b_{1}=b_{1}+e$, whence $a_{1}=a_{1}+e=a_{1}^{\prime}+d_{1}+e=$ $b_{1}^{\prime}+d_{1}=b_{1}$. Similarly, we deduce that $a_{1}=b_{1}$ if $b_{1}^{\prime} \leq a_{1}^{\prime}$. This means that $c_{2}+\left(c_{3}+\cdots+c_{n}+a\right)=c_{2}+\left(c_{3}+\cdots+c_{n}+b\right)$. By iteration of this process, we get $a=b$. Therefore, $M$ is separative, which concludes the proof.

Corollary 12. Let $N$ be a refinement extension of a refinement monoid $M$. If $N$ contains an order-unit $u$, then the following are equivalent:

(1) $M$ is separative. 
(2) $(\forall a, b \in N)(\forall c \in M)(c+a=c+b \leq u$ with $c \propto a, b \Longrightarrow a \leq b$ or $b \leq a)$.

Proof. $(2) \Rightarrow$ (1) follows from Theorem 11 .

(1) $\Rightarrow(2)$ Suppose that $c+a=c+b \leq u$ and $c \in M, c \propto a, b$. Then we may choose $n \in \mathbb{N}$ such that $c \leq n a, n b$. Thus we have $d \in N$ such that $c+d=a+(n-1) a$. Analogously to Corollary 8 , there are refinement matrices over $N$ :

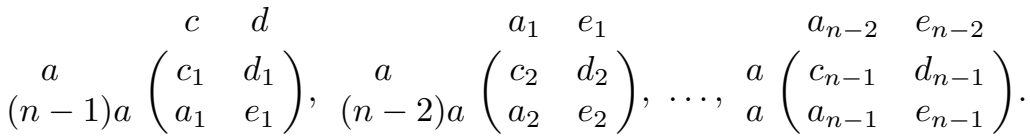

Let $c_{n}=a_{n-1}$. Then $c=c_{1}+c_{2}+\cdots c_{n}$ with $c_{1}, \ldots, c_{n} \leq a$. Similarly, we prove that $c_{1}=c_{11}+\cdots+c_{1 m_{1}}$ with $c_{1 j} \leq a, b\left(j=1, \ldots, m_{1}\right)$. Analogously, we have $c_{i j} \in M$ such that $c_{i}=c_{i 1}+\cdots+c_{i m_{i}}$ and $c_{i j} \leq a, b(i=2, \ldots, n, j=$ $\left.1, \ldots, m_{i}\right)$. This implies that $\left(\sum_{1 \leq i \leq n, 1<j \leq m_{i}} c_{i j}\right)+a=\left(\sum_{1 \leq i \leq n, 1<j \leq m_{i}} c_{i j}\right)+b$ with all $c_{i j} \in M, c_{i j} \leq a, b$. Using Theorem 7 repeatedly, we conclude that $a=b$, as desired.

Theorem 13. Let $N$ be a refinement extension of a refinement monoid $M$. If $N$ contains an order-unit $u$, then the following are equivalent:

(1) $M$ is separative.

(2) $(\forall a, b \in M)(2 a=a+b=2 b \leq u \Longrightarrow a=b$.

(3) $(\forall a, b \in M)(2 a=a+b=2 b \leq u \Longrightarrow a \leq b$ or $b \leq a)$.

Proof. (1) $\Rightarrow(2)$ and $(2) \Rightarrow(3)$ are trivial.

$(3) \Rightarrow(1)$ Given $c+a=c+b \leq u$ with $c \leq a, b$ and $a, b, c \in M$, it follows by [3, Lemma 2.7] that there is a refinement matrix over $\left.M: \begin{array}{cc}c & c \\ b & a_{1} \\ b_{1} & d\end{array}\right)$ with $c_{1} \leq a_{1}, b_{1}$. So we can find $x, y \in M$ such that $a_{1}=c_{1}+x$ and $b_{1}=c_{1}+y$, and then $2 c_{1}+x=c_{1}+a_{1}=c=c_{1}+b_{1}=2 c_{1}+y$. This implies that $2\left(c_{1}+x\right)=\left(c_{1}+x\right)+\left(c_{1}+y\right)=2\left(c_{1}+y\right)=a_{1}+b_{1} \leq a+c \leq u$. Вy hypothesis, we get $a_{1} \leq b_{1}$ or $b_{1} \leq a_{1}$. As a result, $a=a_{1}+d \leq b_{1}+d=b$ or $b=b_{1}+d \leq a_{1}+d=a$, and therefore the proof is true by Theorem 11 .

Corollary 14. Let $I$ be an exchange ideal of a ring $R$. Then the following are equivalent:

(1) I is separative.

(2) For any $A, B, C \in F P(I), A \oplus C \cong B \oplus C \lesssim^{\oplus} R$ with $C \lesssim \oplus A, B \Longrightarrow$ $A \lesssim^{\oplus} B$ or $B \lesssim^{\oplus} A$.

(2) For any $A, B \in F P(I), 2 A \cong A \oplus B \cong 2 B \lesssim \oplus \Longrightarrow A \lesssim \oplus B$ or $B \lesssim^{\oplus} A$.

Proof. In view of Lemma $4, V(I)$ is a refinement monoid. Let $F P(R)$ denote the class of finitely generated projective right $R$-modules, and let $V(R)$ be the monoid of isomorphism classes of objects from $F P(R)$. Then $V(I)$ is a 
submonoid of $V(R)$. Furthermore, we prove that $V(R)$ is a refinement extension of the refinement monoid $V(I)$ and $V(R)$ contains an order-unit $[R]$. Therefore we complete the proof by Theorem 11 and Theorem 13 .

Recall that a ring $R$ is regular provided that for every $a \in R$ there exists $x \in R$ such that $a=a x a$. We say that $a \in R$ is one-sided unit-regular if there exists a right or left invertible $u \in R$ such that $a=a u a$. We write $r(a)$ and $\ell(a)$ for the right and left annihilators of $a \in R$. In [3, Proposition 6.2], Ara et al. proved that a regular ring $R$ is separative if and only if each $a \in R$ satisfying $\operatorname{Rr}(a)=\ell(a) R=R(1-a) R$ is unit-regular. We generalize this result as follows.

Corollary 15. Let $I$ be an ideal of a regular ring $R$. Then the following are equivalent:

(1) I is separative.

(2) Each $a \in R$ satisfying $\operatorname{RaR} R(1-a) R \subseteq R r(a) \bigcap \ell(a) R \bigcap I$ is onesided unit-regular.

(3) Each $a \in R$ satisfying $\operatorname{Rr}(a)=\ell(a) R=R(1-a) R \subseteq I$ is one-sided unit-regular.

Proof. $(1) \Rightarrow(2)$ Suppose $R a R \bigcap R(1-a) R \subseteq R r(a) \bigcap \ell(a) R \bigcap I$. Then $R=$ $(r(a) \oplus r(1-a)) \oplus B$ for a right $R$-module $B$, and so $a R=\operatorname{ar}(1-a)+a B=$ $\operatorname{ar}(1-a) \oplus a B$. Assume that $a=a c a$ for a $c \in R$. Then $R=r(a) \oplus r(1-a) \oplus B=$ $(1-a c) R \oplus r(1-a) \oplus a B$. This yields $r(a) \oplus B \cong(1-a c) R \oplus a B$ with $B \cong a B$. Let $\varphi: a B \rightarrow(1-a) a B$ given by $\varphi(a r)=(1-a)$ ar for any $r \in B$. It is easy to verify that $\varphi$ is a right $R$-module isomorphism, and so $B \cong a(1-a) B=a(1-a) R$. As $a(1-a) R \subseteq R a R \bigcap R(1-a) R \subseteq R r(a)$, it follows by [5, Corollary 2.23] that $B \lesssim m r(a)$ for some $m \in \mathbb{N}$. As $a(1-a) R \subseteq R a R \bigcap R(1-a) R \subseteq$ $\ell(a) R=R(1-a c) R$. By [5, Corollary 2.23] again, $B \lesssim^{\oplus} n(1-a c) R$ for some $n \in \mathbb{N}$. Since $a(1-a) \in I$, we see that $B \in F P(I)$. Let $\mathcal{M}_{R}$ denote the class of all right $R$-modules, and let $W(R)$ be the monoid of isomorphism classes of objects from $\mathcal{M}_{R}$. Analogously to Theorem 9 , we prove that $W(R)$ is a refinement extension of the refinement monoid $V(I)$. By Corollary 8, we get $r(a) \cong(1-a c) R \cong R / a R$. This implies that $a \in R$ is unit-regular, as required.

$(2) \Rightarrow(3)$ is obvious.

(3) $\Rightarrow(1)$ Suppose $A \oplus C \cong B \oplus C \lesssim^{\oplus} R$ and $C \lesssim^{\oplus} A, B$ for some $A, B, C \in$ $F P(I)$. Write $R=A_{1} \oplus C_{1} \oplus D=A_{2} \oplus C_{2} \oplus D$, where $A_{1} \cong A, C_{1} \cong C \cong C_{2}$ and $A_{2} \cong B$. Let $a \in R$ induce an endomorphism of $R_{R}$, which is zero on $A_{1}$, an isomorphism from $C_{1}$ onto $C_{2}$, and the identity on $D$. Then $(1-a) R=$ $(1-a)\left(A_{1} \oplus C_{1}\right)$; hence, $a \in 1+I$. Let $\varphi: A_{1} \oplus C_{1} \rightarrow(1-a)\left(A_{1} \oplus C_{1}\right)$ be a right $R$-module given by $\varphi(x)=(1-a) x$ for any $x \in A_{1} \oplus C_{1}$. Since $(1-a)\left(A_{1} \oplus C_{1}\right)$ is a projective right $R$-module, $(1-a) R \lesssim^{\oplus} A_{1} \oplus C_{1} \lesssim \oplus 2 A_{1}=2 r(a)$. By [5, Corollary 2.23], $(1-a) R \subseteq \operatorname{Rr}(a)$. This yields $R(1-a) R=\operatorname{Rr}(a)$. Assume that $a=a c a$ for a $c \in R$. Then $(1-a) R \lesssim \oplus A_{1} \oplus C_{1} \cong A_{2} \oplus C_{2} \lesssim \oplus$ $2 A_{2}=2(R / a R) \cong 2(1-a c) R$. Using [5, Corollary 2.23] again, $(1-a) R \subseteq$ 
$R(1-a c) R=\ell(a) R$, and then $R(1-a) R=\ell(a) R$. By assumption, $a \in R$ is one-sided unit-regular. This shows that $r(a) \lesssim^{\oplus} R / a R$ or $R / a R \lesssim^{\oplus} r(a)$. Thus we have either $A \lesssim \oplus B$ or $B \lesssim \oplus$. According to Corollary 14, we complete the proof.

As is well known, every one-sided unit-regular ring is separative. It follows from Corollary 15 that a regular ring $R$ is separative if and only if each $a \in R$ satisfying $\operatorname{RaR}(1-a) R \subseteq \operatorname{Rr}(a) \ell(a) R$ is one-sided unit-regular.

Acknowledgements. The author would like to thank the referee for his/her corrections, which lead to the new version of this paper.

\section{References}

[1] P. Ara, Extensions of exchange rings, J. Algebra 197 (1997), no. 2, 409-423.

[2] _ Stability properties of exchange rings, International Symposium on Ring Theory (Kyongju, 1999), 23-42, Trends Math., Birkhauser Boston, Boston, MA, 2001.

[3] P. Ara, K. R. Goodearl, K. C. O'Meara, and E. Pardo, Separative cancellation for projective modules over exchange rings, Israel J. Math. 105 (1998), 105-137.

[4] G. Brookfield, Monoids and Categories of Noetherian Modules, Ph. D. Thesis, University of California, 1997.

[5] K. R. Goodearl, Von Neumann Regular Rings, Monographs and Studies in Mathematics, 4. Pitman (Advanced Publishing Program), Boston, Mass.-London, 1979.

[6] _ Von Neumann regular rings and direct sum decomposition problems, Abelian groups and modules (Padova, 1994), 249-255, Math. Appl., 343, Kluwer Acad. Publ., Dordrecht, 1995.

[7] E. Pardo, Comparability, separativity, and exchange rings, Comm. Algebra 24 (1996), no. 9, 2915-2929.

[8] F. Wehrung, Restricted injectivity, transfer property and decompositions of separative positively ordered monoids, Comm. Algebra 22 (1994), no. 5, 1747-1781.

Department of Mathematics

HANGZHOU NORMAL UNIVERSITY

HANGzHou 310036, China

E-mail address: huanyinchen@yahoo.cn 